\title{
Aproximación histórica al concepto de experiencia estética a propósito del diálogo entre la literatura y la enseñanza de las ciencias en la universidad
}

\author{
Historical approach to the concept of aesthetic experience concerning \\ the dialogue between literature and the teaching of sciences at the university
}

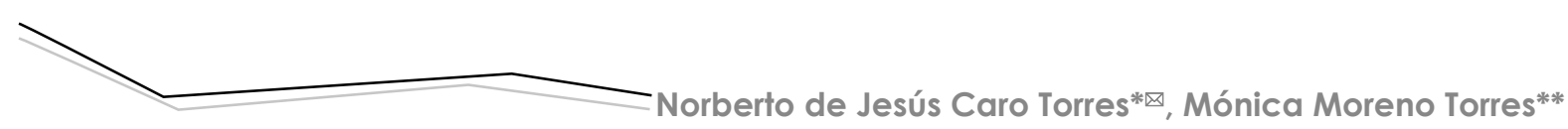

Caro Torres, N. J., \& Moreno Torres, M. (2017). Aproximación histórica al concepto de experiencia estética a propósito del diálogo entre la literatura y la enseñanza de las ciencias en la universidad. Investigación y Ciencia de la Universidad Autónoma de Aguascalientes, 25(72), 82-92.

\section{RESUMEN}

El texto responde a las preguntas: ¿Cómo surge el concepto de experiencia estética en la obra literaria?, ¿cómo se puede dar el diálogo de saberes entre la literatura y la enseñanza de las ciencias en la universidad? Por ello, parte de una reflexión en torno al hipertexto y la literatura, y aborda el pensamiento de algunos autores clásicos del siglo XVIII y principios del XIX, quienes tuvieron como preocupación principal el tema del arte. Estas reflexiones comienzan a mostrar que la didáctica universitaria debe considerar la relación entre la enseñanza de las ciencias y la estética, como posibilidad para otorgar al futuro profesional una visión holística de su ser y del mundo.

Palabras clave: experiencia estética; didáctica universitaria; enseñanza de las ciencias; literatura; ciencia; estrategia didáctica.

Keywords: aesthetic experience; higher education teaching methods; teaching of sciences; literature; science; didactic strategy.

Recibido: 21 de enero de 2017, aceptado: 4 de septiembre de 2017

* Departamento de Educación Infantil, Facultad de Educación, Universidad de Antioquia. Calle 67 No. 53-108, Medellín, Antioquia, Colombia. Correo electrónico: norberto.caro.torres@gmail.com

** Centro de Investigaciones Pedagógicas-CIEP, Facultad de Educación, Universidad de Antioquia. Calle 67 No. 53-108, Medellín, Antioquia, Colombia. Correo electrónico: monica.moreno@udea.edu.co

Este texto hace parte de los resultados parciales de la investigación "Diálogos de la literatura con la enseñanza de las ciencias en la universidad: Construcción de una estrategia didáctica basada en una experiencia estética con el cuento y la novela", adelantada por Norberto de Jesús Caro Torres para su tesis en el Doctorado en Educación de la Universidad de Antioquia, y asesorado por la doctora Mónica Moreno Torres.

$\bowtie \quad$ Autor para correspondencia
Esta intención comienza a reflejarse en los resultados parciales del diseño y aplicación de una serie de talleres que conducían a la elaboración de un ensayo referido a la formación profesional de los estudiantes de la Escuela de Nutrición y Dietética y de la Facultad de Medicina, ambas unidades académicas de la Universidad de Antioquia, Colombia. Al final del artículo, las conclusiones muestran las posibilidades que brinda la estrategia didáctica en proceso al diálogo de saberes entre la literatura, la ciencia y la didáctica universitaria.

\section{ABSTRACT}

This text is the answer to these questions: $\dot{2}$ How the aesthetic experience come about in the literary work?, zhow to reach a dialogue of wisdoms between literature and the teaching of sciences at the university? Because of that, it starts from a reflection around hypertext and literature, and it addresses the thinking of some classic authors of the XVIII and the beginning of the XIX century, who had as a main concern the art topic. These considerations start to show that higher education teaching methods must consider the relation between the teaching of sciences and aesthetics, as a possibility of granting to the future college graduates a more holistic vision of his being and the world. This intent begins to reflect on the partial results of the design and the application of a set of assignments that drove to the elaboration of an essay referred to the professional formation of the students in the School of Nutrition and Dietetics and the Medical Faculty, both are academic units of The University of Antioquia, Colombia. At the end of the article, the conclusions show the possibilities that the didactic strategy in process provides, to the dialogue 
of wisdoms between literature, science and higher education teaching methods.

INTRODUCCIÓN

En 1963, con la publicación de la novela Rayuela del escritor argentino Julio Cortázar, se vislumbraron y anticiparon revoluciones en el orden científico, técnico, tecnológico, cultural, intercultural y multicultural. Este escritor, con homólogos como Borges (2000), Sábato (1911-2011), Bioy Casares (19141999) y Leopoldo Lugones (1874-1938), así como la teoría crítica del hipertexto sustentada por Landow (1995), han posibilitado la convergencia de dos campos del saber sin ninguna conexión aparente, como son las teorías literarias y el hipertexto. Según Landow, se estaba produciendo un cambio de paradigma en los escritos de Jacques Derrida, Theodor Nelson, Roland Barthes y Andries van Dam. A este giro se unen otros especialistas en hipertexto: Argos y Ezquerra (2013), lanni (2006), Rueda (2007), Rueda Ortiz y Quintana Ramírez (2007) y Vargas Guillén (1998), quienes abandonaron los sistemas conceptuales basados en nociones como centro, margen, jerarquía y linealidad, y los sustituyeron por otros, como multilinealidad, nodos, nexos y redes.

Barthes en S/Z (1984) describe un ideal de textualidad que coincide con lo que se conoce como hipertexto electrónico. De manera similar, la novela Rayuela constituye un hipertexto, en cuanto tiene características hipermediales, pues sus tramas y discursos se pueden leer de manera lineal o discontinua. Esta novela sugiere signos y símbolos

Según Pablo Capanna (1966, p. 7), el concepto de ciencia ficción viene del norteamericano Gernsback, quien en 1926 designó el nuevo género comercial-literario como scientifiction o science fiction o s-f. Este concepto alude a un tipo de fantasía que tomaba como tema la ciencia, los científicos y el método; sin embargo, de acuerdo con este autor, la evolución posterior de las s-f se desarrolló bajo esta marca inadecuada. Posteriormente, en Francia se impuso science fiction, en Norteamérica igual que en Alemania fantasy fiction, en Italia fantascienza y en España fantaciencia. En Francia se ha hablado de novela de anticipación desde Julio Verne, además de ciencia novelada, anticipación científica y humor científico. Pierre Versins propuso subsumir este género en el campo de las "literaturas conjeturales"; a su vez, Bergier y Pauwels propusieron el término literatura diferente. Por su parte, algunos anglosajones propusieron los términos science fantasy (William Tenn), speculative fantasy (Michael Moorcok), ficción especulativa (Damon Knight) (citados por Capanna, 1966, pp. 10-12).

2 Desde la perspectiva de Carl Djerassi (2011), escritor, científico e inventor de la píldora anticonceptiva, ciencia en ficción es aquella producción literaria en la cual la trama gira en torno a los problemas cruciales y cotidianos de la ciencia real, y la mayoría de los personajes son científicos, tanto reales como imaginarios.

3 En términos de Garrido (2011, p.18) el ámbito de la literatura es el de la invención, la creación de mundos y la ficción, entendida como mímesis que deviene de la concepción aristotélica. relacionados con las ciencias, tales como límite, infinito, circularidad, caos, la naturaleza química de los procesos mentales y la fragmentación de la existencia, entre otros. Estos elementos están presentes en la enseñanza de diversas ciencias como la matemática, la física y la psicología, entre otras.

Algunos enfoques contemporáneos en la enseñanza de las ciencias (Cachapuz, 2007; García García \& González Agudelo, 2007; Gianetti, 2002; Henao, 2014; Maturana \& Varela, 1998; Montes, 1999; Moreno Torres \& Carvajal Córdoba, 2015; Téllez, 2004) señalan la importancia de brindar a los estudiantes y sus profesores una educación estética que, preocupada por su formación ética, política y académica, reconozca en la recepción estética del cuento y la novela un proceso de mediación capaz de ampliar el horizonte conceptual y vivencial de dichos sujetos. Por su parte, algunos autores de áreas diferentes de la literatura han estudiado dicha relación y hasta han llegado a convertirla en objeto de creación en su calidad de científicos, como Herbert George Wells (1866-1946), Aldous Huxley (1979); Julio Verne (1828-1905); Isaac Asimov (1920-1994), entre otros. Sus propuestas literarias poco se han estudiado en el ámbito universitario, sobre todo, desde el campo de las ciencias básicas. Así que enseñar ciencias desde una visión humanista y estética exige reconocer la importancia que tiene la formación de profesionales en varias direcciones. La lectura que estas personas realicen de los textos científicos no quedaría por fuera de la experiencia estética que los mismos podrían tener con textos literarios, bien sea de ciencia ficción', ciencia en ficción ${ }^{2}$ o de ficción ${ }^{3}$. De ahí que resulte de vital importancia reabrir la reflexión acerca de la relación o el vínculo entre la literatura y la enseñanza de las ciencias, esto es, volver sobre una eterna simbiosis entre el arte y la ciencia, establecida hasta cierto momento de la historia pero que en vista de la necesidad imperante de especializar los discursos se han ido separando y bifurcando a la largo de los siglos XX y XXI.

Por otra parte, cuando se estudia la historia del concepto experiencia estética en la obra literaria, se puede observar que las concepciones sobre las cuales se sustenta no están por fuera de una filosofía del ser humano, necesaria para la formación de las futuras generaciones de profesionales; lo que en términos de Leibrandt (2016) permitiría avanzar, contribuir en su formación estético emocional, porque la literatura aporta significativamente en la manera en que los sujetos perciben y se relacionan con el mundo. 
Asimismo, busca la formación de una competencia literaria y fomenta las emociones, la imaginación y la participación subjetiva, imprescindibles en una experiencia estética. Pero también en palabras de la misma escritora (Leibrandt, 2012) la literatura, las narraciones que incitan a la búsqueda de la propia identidad pueden despertar el interés y el deseo de conocer más acerca del pasado y se puede construir la conciencia histórica desde la infancia a través de las obras literarias. Avanzar en esta perspectiva podría permitir a la didáctica universitaria convertirse en un medio para el docente de la educación superior.

\section{Recorrido histórico de la experiencia estética en la obra literaria}

Entre los siglos XVIII y XIX, Alexander Gottlieb Baumgarten en Reflexiones filosóficas acerca de la poesía (1975), Karl Philip Moritz en Propuesta para la unificación de todas las bellas artes y las ciencias bajo el concepto de lo perfecto en sí mismo (2004) e Immanuel Kant en Crítica del juicio (2010) fueron trascendentales, pues tomaron fuerza las concepciones sobre autonomía del arte en consonancia con las teorías del arte por el arte (Aguiar e Silva, 1986), al analizar la finalidad de este en la sociedad. Así, Baumgarten, creador del vocablo estética en su obra Reflexiones filosóficas acerca de la poesía, cuya primera edición salió en 1735, entiende por poesía el discurso sensible perfecto; denomina poética el complejo de reglas al que aquella se conforma; filosofía poética, el hábito o disposición de componer el poema; y poeta, el hombre que goza con esta inclinación (1975).

Según Cabot (1999), es Baumgarten quien utiliza por primera vez el término estética (en 1735) referido a una determinada disciplina filosófica, en el sentido de una teoría del conocimiento sensible en general y de su forma específica: el gusto. Para este autor, el término estética se difundió como nombre de una nueva especialidad o disciplina, y sufrió progresivos cambios de significado hasta Kant. Sin embargo, para María Antonia Labrada (1983) el problema fundamental no radica en el hecho de que en la obra de Baumgarten aparezca por primera vez el término aesthetica. Es decir, si la definición que da de la estética como "ciencia del conocimiento sensitivo" ha pasado inadvertida en ocasiones, es por la falta de contextualización en el terreno gnoseológico que le corresponde.

Baumgarten define las diferentes partes del poema como representaciones sensibles, y el nexo entre estas, y las palabras como signos suyos; asimismo, precisa lo poético como todo lo que puede contribuir a la perfección del poema. Igualmente, plantea que las representaciones sensibles que pueden ser oscuras o claras constituyen las partes del poema; es decir, las representaciones de una misma cosa pueden ser oscuras para A, claras para $\mathrm{B}$ o distintas para $\mathrm{C}$. Baumgarten considera que las representaciones oscuras no contienen las suficientes representaciones de cosas conocidas como para poder reconocer y distinguir lo representado de todo lo demás, pero ellas están contenidas en las representaciones claras. De esta manera, al poseer esta virtud, proporcionarán mayores elementos para dar a conocer las representaciones sensibles como si fuesen oscuras. Además, considera que entre más claramente se representen las imágenes, más semejantes se harán las impresiones sensibles, hasta el punto de ser equivalentes con alguna frecuencia a una sensación más débil. Es poético representar imágenes lo más claramente posible, pero también lo es hacerlas muy semejantes a las sensaciones. Esto lo lleva a proponer que un poema y una pintura son cosas bastante semejantes. En este punto, los pone en el lugar de la semejanza no desde lo artístico, sino en razón del efecto alcanzado; esto es, la sensación que producen el arte y la poesía en el perceptor. En este contexto propone: "La poesía será como una pintura" (1975, p. 52). Igualmente, plantea que un poema es más perfecto que una pintura en tanto representación de cosas sensibles.

Aquí se pueden considerar dos aspectos: por un lado, el referido a la perfección del poema en relación con la pintura; y por otro, la importancia del carácter sensible del poema. En esta perspectiva, hay un intento por desentrañar el concepto de sensibilidad en relación con la experiencia estética del perceptor, pues una vez que este percibe el poema se abren múltiples posibilidades a la sensación y a la imaginación. En palabras de Julio del Valle (2011), con Baumgarten la estética nace como un intento por recuperar la particularidad sensible que se había sacrificado en aras de encontrar la verdad, instalada en la lógica; en este sentido, es fundamental la sensibilidad particular, no la universal. Se podría decir que Baumgarten hace un llamado a la experiencia estética del sujeto y abre caminos a pensadores como Iser (2005) y Jauss (1992, 2000, 2002), quienes sustentan posteriormente este concepto en relación con la experiencia literaria, en consonancia con los planteamientos de Larrosa (2003) y Farina (2005). 
Baumgarten, filósofo de la estética, vuelve a la idea de representaciones confusas que provienen de elementos separados y combinados en la imaginación, en tanto imágenes poéticas; pero los objetos de tales representaciones son posibles o imposibles en el mundo real. Él considera verdaderas estas primeras representaciones ficcionales. A su vez, dice que los objetos denotados por las ficciones son imposibles en el mundo real, o imposibles en cualquiera de los mundos posibles. Ahora bien, llama "utópicas" las cosas absolutamente imposibles; las otras son "heterocósmicas" (Dolezel, 1999). Finalmente, Baumgarten señala que solo son poéticas las ficciones verdaderas y heterocósmicas; es decir, aquellas que hacen parte de un mundo posible. En términos de Eco, los mundos posibles están en relación con una construcción cultural (1993). Bruner (1987) establece que tanto la ciencia como la literatura crean posibilidades de existencia a través de los mundos posibles. Para Albaladejo (1996) los modelos de mundo, que pueden ser de tres tipos: tipo I o de lo verdadero, tipo II o de lo ficcional verosímil y de tipo III de lo ficcional no verosímil, son creados por el autor del relato y responden a su decisión y a su relación con el lector.

Baumgarten convierte la palabra en una bisagra encargada de poner en movimiento el discurso científico y el literario. Cuando habla de la incompatibilidad semántica entre el lenguaje de la escuela y el del arte, parece sugerir la disyunción que históricamente ha convertido la enseñanza de las ciencias en un saber desarticulado del arte en sus múltiples expresiones. Esta dificultad pervive en nuestra contemporaneidad, de allí la importancia de incluir en el currículo universitario una propuesta didáctica que dialogue con la visión estética del profesor y la de aquellos sujetos encargados del desarrollo científico y tecnológico de la sociedad.

\section{El arte como microcosmos en Moritz}

Según Aguiar e Silva (1986), fue Karl Philip Moritz en Sobre la imitación plástica de lo bello, cuya edición original salió en 1778, quien propuso que la obra de arte constituye un microcosmos, un todo orgánico, completo y perfecto en sí mismo, y bello precisamente porque no tiene necesidad de ser útil. Desde esta perspectiva, la utilidad se mira como un factor extraño a la belleza, en tanto esta posee en sí misma su valor total y la finalidad de su existencia. En este contexto, Jaime Aspiunza (2010) señala que Moritz comenzó por examinar las nociones de útil, bueno, noble y bello, cuando estas se aplicaban a la condición de un hombre o de una acción. Observó que existía una asociación fundamental entre lo útil y lo bueno, y lo noble y lo bello. Asimismo, explicó la ordenación ascendente de los cuatro conceptos mencionados - útil, bueno, noble, bello-en función de lo que se podría llamar independencia, valor propio; porque el hombre útil atrae la atención en relación con un conjunto de cosas exteriores a él; el bueno, porque se confía en que no va a perturbar la paz de nadie; y solo el noble atrae y admira por sí mismo. En cuanto a lo bello como concepto práctico opone resistencia, porque se diferencia de lo noble como lo exterior de lo interior; es decir, lo noble es una suerte de belleza del alma y en esta misma equiparación queda claro. Aspiunza señala que no es necesario separarlos, porque la belleza exterior es a la vez estampa de la belleza interior del alma, lo que comprende también en sí lo noble (2010).

De esta manera, el objeto meramente útil no es algo acabado o perfecto en sí mismo, deviene una vez lo haya aprehendido el sujeto. Por tanto, lo útil no posee una finalidad en sí, sino fuera de sí; su finalidad es externa y puede ser creada como se quiera. A modo de ejemplo, Moritz afirma que el reloj y el cuchillo tienen su finalidad fuera de sí, puesto que el hombre se sirve de ellos para su comodidad; por ello, no son algo acabado en sí y carecen de valor estricto sin la consecución real o posible de su finalidad externa (2004). En cambio, en lo bello ocurre todo lo contrario: la finalidad no está fuera de sí, ya que existe por su propia perfección interna. En este sentido, lo bello no necesita ser recreado, pero sí necesita ser reconocido por el sujeto. Por ello argumenta que "podemos subsistir muy bien sin la contemplación de bellas obras de arte, estas, sin embargo, no pueden subsistir, al menos como tales, sin nuestra contemplación" (p. 183).

\section{Autonomía del arte en Kant}

En el Prólogo de Crítica del juicio, publicada originalmente en 1790, Kant plantea que la búsqueda de un principio para la naturaleza del juicio se encuentra en los juicios llamados estéticos, pues ellos "se refieren a lo bello y lo sublime de la naturaleza o del arte" (2010, p. 303). El "juicio estético" es una facultad particular de juzgar las cosas según una regla, pero no según conceptos. Entiende gusto como la facultad de juzgar lo bello; y este juicio del gusto no es lógico sino estético, entendido como aquel cuya base determinante es subjetiva. En el $\S$ 2 dice que la satisfacción que determina el juicio del gusto es totalmente desinteresada; así, cuando un 


\section{INVESTIGAGIÓก Y CIECEIA DE LA UNIVERSIDAD AUTÓNOMA DE RGUASCALIERTES}

objeto es bello y se muestra el gusto, este hace una referencia a la autonomía; esto es, lo que el sujeto piensa realmente y por sí mismo del objeto y no los otros sujetos sobre el mismo objeto. Esta idea lo lleva a plantear en el § 3 que la satisfacción en lo agradable está unida al concepto de interés. De esta manera, considera lo agradable como aquello que place a los sentidos en la sensación; pero esta última puede tener una doble significación; es decir, la satisfacción en lo bueno y en el interés.

Kant considera que la satisfacción en lo bello tiene que depender de la reflexión sobre el objeto, la cual conduce a tener cualquier concepto, y así se puede distinguir de lo agradable que descansa en la sensación. En el § 4 propone que lo agradable y lo bueno parecieran tener el mismo valor: "Todo deleite es bueno en sí mismo", porque en lo bueno viene siempre el saber si es solo mediata o inmediatamente bueno -útil o bueno en sí- (p. 337). En este mismo sentido, en el $\S 5$ se centra en lo agradable, lo bello y lo bueno. En estos modos de satisfacción, la del gusto en lo bello es la única desinteresada y libre, pues no hay interés alguno, ni el de los sentidos ni el de la razón, que arranque el aplauso. En estos tres casos de satisfacción están presentes la inclinación y la complacencia o estimación, facilitadoras, en cierta medida, del gusto estético; asimismo, Kant se refiere a lo bello como aquello que place universalmente ( $\S$ 9, p. 349).

En el $\S 17$ analiza el ideal de la belleza, la que precisa como forma de finalidad de un objeto en cuanto este ideal de belleza es percibida en él sin la representación de un fin. Para Kant, lo bello se define como "objeto de una necesaria satisfacción" (§ 22). Esto indica que el gusto es una facultad de juzgar un objeto en relación con la libre conformidad a las leyes de la imaginación (§ 9, p. 369). De esta manera, surge la pregunta por una presunta contradicción cuando se pretende que la imaginación sea libre $y$, al mismo tiempo, responda a unas leyes. Sin embargo, Kant dirime esta dicotomía al decir que solo el entendimiento da la ley; esto es, el conocer y el poder tomar decisiones sobre la obra de arte relativa a la cosa misma, sin que la imaginación sea obligada a proceder según una ley determinada, así se determine por conceptos cómo deba ser, según la forma, su producto. Pero la satisfacción no es la que se da en lo bello, sino en lo bueno, dentro de la perfección formal; y el juicio, agrega, no es un juicio por medio del gusto. Esto significaría poder concebir la libre conformidad del entendimiento basado y de acuerdo con unas leyes que están en relación con la representación que tengamos del objeto bello, y una subjetiva, que está en concordancia con la imaginación y el entendimiento (§ 22, pp. 369-370).

En síntesis, al mostrar la evolución del concepto obra de arte, Kant señala que si el arte fuera una mera sensación -algo subjetivo- debería estar acompañado de placer; entonces, ese producto artístico no placería en el juicio más que por medio del sentimiento sensible. Esto quiere decir que la sensibilidad y la contemplación de la obra de arte no serían suficientes sin una valoración o reflexión sobre la misma. Esta reflexión debe realizarse con base en las "leyes de la imaginación", entendiéndose por esto último como "la facultad de conocer productiva" (2010, § 49, p. 440). Así, la obra de arte se presenta ante el hombre, quien se entretiene con ella cuando la experiencia se hace demasiado superflua, pero la transforma por medio de leyes analógicas, y según principios de la razón - naturaleza empírica- se entrega a ella y siente libertad frente a la ley de asociación que está unida al uso empírico de aquella facultad; por tanto, la naturaleza presta materia y el hombre la arregla para que supere la naturaleza.

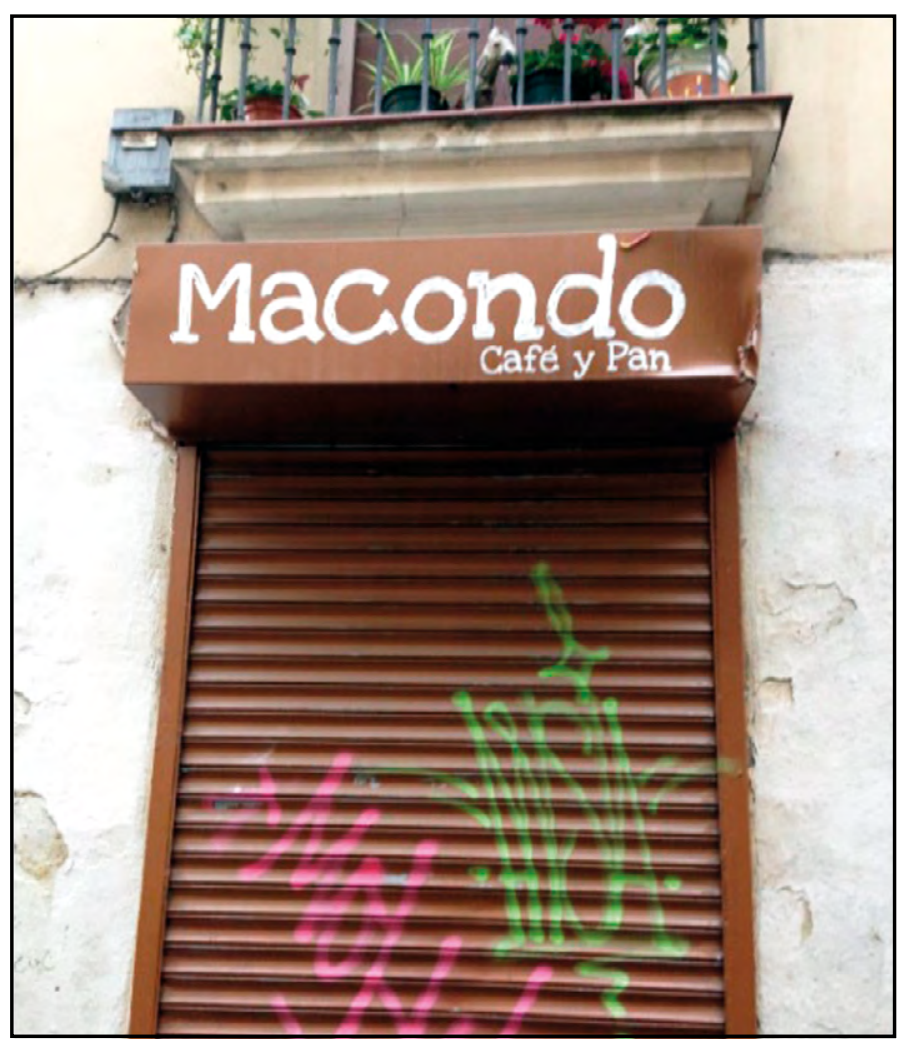

Figura 1. Pórtico en la parte antigua de la ciudad, Navarra, España. Fotografía de Norberto de Jesús Caro Torres. 


\section{MATERIALES Y METODOS}

La investigación está inscrita en el enfoque de la experiencia hermenéutica desde la perspectiva gadameriana, sustentado y desarrollado por González Agudelo (2011). En esta perspectiva, la investigación hermenéutica comprende el todo -experiencia estética- como anticipación de sentidos, y las partes —novela, cuento, enseñanza de las ciencias, didáctica universitaria-como unidades de significación. De esta manera, de cada parte se configura un todo y de este emergen nuevas partes, dando así sentido al círculo de la comprensión. Este círculo incluye la anticipación de sentidos, que tiene como uno de sus propósitos la comprensión del texto desde la historicidad de su contenido en busca de la verdad como una posibilidad, entre otras. Así, la verdad del contenido del texto se establece por medio del diálogo entre las preguntas y las respuestas que surgen del contacto del hermeneuta con el pasado de su objeto de estudio. En este ir hacia atrás para reconocer los rasgos del pasado en el presente, el diálogo con los textos cumple una función importante; esto es, construir un acuerdo en relación con la cosa investigada -experiencia estética-.

Dicha fusión del pasado y el presente de lo investigado tiene un proceder hermenéutico cuyas partes son: el problema dialéctico, la hipótesis abductiva, la historia de conceptos, el estado en cuestión, el acopio de la información, la cosa creada, el acuerdo con la cosa y la unidad de sentido. Este procedimiento incluye: los prejuicios, la reflexión, el análisis, la comparación, la comprensión, la interpretación y la síntesis. Esta es la praccis, que en términos de González Agudelo determina al ser y lo hace partícipe de una comunidad de indagación:

Así, desde las vivencias, donde habitan nuestros prejuicios, nos preguntamos, reflexionamos, analizamos y comparamos para comprender. Comprender no es reproducir el pasado del texto, resumirlo, participar en un significado presente, el significado común, en fusión de horizontes. La comprensión es una apropiación. Pero ninguna comprensión es acabada, nunca se comprende completamente, la comprensión no se cierra al futuro. (...) No hay predominio del ser sobre la cosa investigada, pues la fusión de horizontes no es lineal.

4 La abducción es un tipo de inferencia que se caracteriza por su probabilidad: la conclusión que se alcanza es siempre conjetural, es solo probable, pero al investigador le parece del todo plausible. Es en esa plausibilidad, en ese carácter intuitivo donde radica su validez, y no en su efectiva probabilidad que tiene solo una influencia indirecta (Peirce en Nubiola, 2001, p. 5).
Se avanza mirando el pasado y se retrocede, reconociendo en la historia de los conceptos la proyección de sentido del problema dialéctico y la hipótesis abductiva. (González Agudelo, 2011 , pp.129 y 141)

En consonancia con lo anterior, la pregunta de investigación es la siguiente: ¿ Cómo una experiencia estética con el cuento y la novela permite a un grupo de estudiantes de educación superior identificar el diálogo entre la literatura y las ciencias? La hipótesis abductiva $^{4}$ se formula a partir de la siguiente pregunta: ¿ Una estrategia didáctica fundamentada en la experiencia estética con el cuento y la novela podría contribuir al diálogo entre la literatura y la didáctica universitaria?

La pregunta se dirige a los perceptores de la obra literaria, considerados como interlocutores válidos para avanzar en el diseño, desarrollo y evaluación de la estrategia didáctica en proceso de elaboración. La hipótesis abductiva considera que la formación de profesionales en diversas áreas de la universidad podría incluir, en dichos sujetos y sus profesores, la experiencia estética como un proceso de mediación para el desarrollo de la didáctica universitaria. En síntesis, el proceder hermenéutico se convierte en el punto de partida para avanzar en el diseño de los instrumentos de esta investigación. Para el efecto, a continuación se presentan los resultados de los ensayos de los estudiantes de la Escuela de Nutrición y Dietética y de la Facultad de Medicina de la Universidad de Antioquia.

\section{RESULTADOS}

Parte de la investigación se llevó a cabo con estudiantes de primer semestre de la Escuela de Nutrición y Dietética de la Universidad de Antioquia en el semestre 2015-II y estudiantes de Medicina de la Facultad de Medicina del segundo semestre de la misma Universidad en el periodo 2016-II. El trabajo final de los cursos Socialización del conocimiento y Comunicación II, consistía en la elaboración de un ensayo en el cual se diera cuenta de lo aprendido durante el semestre académico. Para ello los estudiantes de Nutrición y Dietética en la medida en que el curso avanzaba en temas de la comunicación, iban leyendo el texto de la escritora mexicana Laura Esquivel (1993): Como agua para chocolate. En clase se abordaban temas tratados allí relativos a su campo profesional: alimentos, cuestiones alimentarias, componentes químicos, nutricionales y sus vínculos con el mundo de la vida, sus percepciones estéticas, 
lógicas, políticas y éticas en relación con las vivencias evidenciadas en el texto de Esquivel.

Finalmente, en consenso postularon una serie de temas para realizar la producción textual y la propuesta ganadora fue el tema del vino. Se dieron a la tarea de indagar, buscar y dar comienzo al texto. En esta misma lógica, los estudiantes de Medicina habían trabajado con el profesor titular el tema de las emociones y se les propuso leer el texto del escritor colombiano Gabriel García Márquez (1994): Del amor y otros demonios. Los estudiantes avanzaban en la lectura del texto y en la clase se realimentaba el proceso leyendo en voz alta y haciendo comentarios alrededor de la trama, la enfermedad, las emociones de los personajes, el panorama histórico en el cual se desarrolla la historia, la política, la ética, la lógica y la estética vista en el texto y la manera en que esos personajes con sus experiencias iban siendo parte de sus vidas. De esta forma, los ensayos se fueron configurando, construyendo, en la medida en que se realizaban una serie de talleres con el fin de consolidar la propuesta y el ensayo final.

\section{DISCUSIÓN}

Según la revisión de ensayos elaborados por los estudiantes mencionados, 15 de Medicina y ocho de Nutrición y Dietética, se derivan como resultados parciales los siguientes:

Es importante destacar como generalidades en la construcción de los textos, que en su mayoría los ensayos del programa Nutrición y Dietética sustentan sus argumentos en una más amplia referenciación de fuentes bibliográficas, y en esta misma secuencia los primeros presentan un mayor manejo de la referenciación en cuanto a la cita y bibliografía dentro del texto.

En el caso del Programa de Nutrición y Dietética solo una persona entre ocho no utiliza la citación de alguna fuente bibliográfica, las demás citan no sólo el texto de referencia para el ensayo, sino también otras diferentes fuentes; mientras en el Programa de Medicina sólo siete de 26 estudiantes hacen referencia a alguna fuente de información bibliográfica, en dos de los siete casos sólo referencian como fuente bibliográfica el texto base de lectura para la construcción del ensayo.

A continuación se presenta de manera general lo señalado en los diferentes ensayos. En el Programa de Nutrición y Dietética en relación con el vino y con el texto Como agua para Chocolate de la escritora Laura Esquivel; mientras para el Programa de Medicina el texto Del amor y otros demonios del escritor Gabriel García Márquez, así como La inteligencia Emocional, del escritor Daniel Goleman (1996).

Se realizó la lectura de información teniendo en cuenta tres categorías. En la primera, Relación campo especifico profesional, se mencionan algunos fragmentos dentro de los ensayos que se vinculan con el campo o área específica profesional, como argumentos importantes dentro de sus trabajos; la segunda: Relación entre literatura y ciencia, con el fin de señalar algunos ejemplos de dicha relación, o argumentos para afirmar o negarla; finalmente la tercera categoría: Relación con las emociones, donde se destacan vínculos con el campo personal y de la vida.

\section{Categoría 1: Relación campo específico profesional}

En el Programa Nutrición y Dietética en esta primera relación se destaca un componente histórico del origen del vino que más adelante en otras categorías subraya una fuerte relación con la religión y con el consumo de clases socioeconómicamente altas. Se enfatiza en algunos beneficios del consumo de este producto, del consumo como tradición y de la necesidad de fortalecer la investigación del mismo en Colombia. Este programa enfatiza fuertemente en la composición del vino, sus beneficios en el campo de la salud, específicamente de la alimentación.

Para el segundo grupo, estudiantes de Medicina, los textos son puestos al servicio de la medicina para hacer mención de las enfermedades mentales, a la explicación de ciertos comportamientos de los personajes del texto, lo desencadenado en el cuerpo - el campo de la salud en relación con algunos sentimientos. El amor y otras emociones derivadas también de la música, son tomados en cuenta como elementos estimulantes y positivos para el cuerpo y para desencadenar otras sensaciones de bienestar en este. Es significativo el tratamiento del tema del amor en este grupo, hay una constante valoración del término en términos científicos, y de manera directa en afectaciones positivas para el ser humano. En tanto lo positivo como lo mencionado anteriormente, y en la parte negativa, también desencadenando otros sentimientos alrededor, por ejemplo, de los celos. 


\section{IIVESTIGAGIÓn Y CUERCIA DE LA UחIVERSIDAD AUTÓNOMA DE AGUASCALIERTES}

\section{Categoría 2: Relación entre literatura y ciencia}

En esta categoría se destaca en el primer grupo la relación del vino en el campo religioso y desde los aportes científicos. Se vincula el vino con algunas obras, y en este sentido se atribuye un significado al consumo del vino de acuerdo con un contexto histórico, social, religioso, como se ha venido mencionando también en la anterior categoría. Para el segundo grupo, estudiantes de Medicina, hay más información por el mayor número de ensayos, aquí también hay una fuerte relación entre literatura, ciencia y religión.

La relación entre ciencia y religión se percibe como un vínculo estrecho debido a que ambas han buscado explicaciones a diversos cuestionamientos, pero también hay una crítica en algunos ensayos a la religión porque históricamente esta ha limitado los avances científicos. Hay una constante explicación científica de los hechos relatados en los diferentes textos, y una valoración en la relación entre literatura y ciencia como un aporte de la primera para guardar en la historia relatos sobre avances científicos, observar en la historia el campo de la medicina.

\section{Categoría 3: Relación con las emociones}

Para el primer grupo respondiendo al tema de los ensayos el consumo del vino es asociado con un estilo de vida de una población en condiciones socioeconómicamente favorables y, por ende, a la generación de cierto estatus. Para el segundo grupo, como se ha mencionado, ante el mayor número de ensayos y de información se destacan más conclusiones. Hay una recurrencia a la explicación científica de las relaciones afectivas y lo que ellas derivan, y a las emociones.

\section{CONCLUSIONES}

En la categoría 1 de la relación campo específico profesional en el programa de Nutrición y Dietética, existen cuatro subcategorías: la relación del consumo del vino como beneficio, el consumo como tradición, apuestas a la investigación en Colombia, componentes y origen del vino. En esta misma categoría en el programa de Medicina, emergen también cuatro subcategorías, que son las atribuciones y explicaciones del amor y la música en el campo de la salud, atribuciones positivas al desarrollo de la inteligencia emocional en el campo de la salud, aportes de la literatura en el campo de la salud y la relación ciencia y religión. Se puede observar claramente cómo la literatura emerge con mayor claridad en los escritos y en las posturas críticas de los estudiantes de Medicina, mientras que en los estudiantes de Nutrición en esta primera categoría hay una mayor timidez al referirse a la literatura, dado que el tema, si bien es cierto que en las obras de la Antigüedad, Edad Media, Renacimiento (Homero, la llíada y la Odisea; los diálogos platónicos; La divina comedia de Dante Alighieri; las obras cervantinas; las obras picarescas de la España renacentista, entre otras) el vino es un referente importante en la familia, la sociedad y la cultura. Por otro lado, en la obra leída de Esquivel, Como agua para chocolate, a pesar de haber hecho un rastreo previo de cómo la autora aborda el tema del vino, es poco mencionado por ellos; mientras que en Medicina, el texto leído y vivido fue Del amor y otros demonios de García Márquez, sí había una referencia constante muy importante para todos los ensayos dado que el tema eran las emociones.

En la categoría 2, de la relación entre literatura y ciencia, en los estudiantes de Nutrición y Dietética se dan tres subcategorías: relación con el campo religioso, relación especial con la literatura y el vínculo con otras obras. En Medicina emergen otras tres subcategorías: ciencia y religión en búsqueda de explicaciones, explicaciones desde el campo científico y aportes en la relación ciencia y literatura. Se puede inferir que los estudiantes de ambos programas académicos se van acercando al establecimiento de relaciones intertextuales y de diálogo de saberes, entre lo que ellos, desde el campo de la ciencia han estudiado y conocen, con las ciencias sociales y humanas como la religión y la literatura.

Es dable decir que desde la praccis (González Agudelo, 2011), se parte de unos prejuicios que habitan a los interesados; en este caso, los estudiantes indagaron, buscaron y construyeron sus ensayos tratando de encontrar pistas que los llevaran a dar solución a preguntas desde su ser y hacer como profesionales en formación con prejuicios e ideas que se fueron configurando para dar firmeza y textura a las mismas. La construcción de los textos se hizo con base en unos acuerdos al interior de los cursos y basados en referentes científicos y literarios. Los cientíicos fueron aquellos textos que sustentaran teóricamente las bases de datos y otros que les fueron llevados a las clases tanto sobre el vino como sobre las emociones e investigaciones que se han hecho alrededor de las obras de Esquivel en el campo de los alimentos, la cocina, la tradición culinaria, las relaciones políticas 
IIVESTIGACIÓn Y CIERGIA DE LA UחIVERSIDAD AUTÓnOMH DE RGUASCALIERTES y sociales en la época de la Revolución mexicana, la mujer, el machismo, incluyendo la película que lleva su nombre, del director Alfonso Arau (1992).

En el caso de los estudiantes de Medicina, hicieron lo propio con diferentes textos, fruto de investigaciones que se habían realizado sobre la obra de García Márquez en relación con la medicina, las emociones, las condiciones políticas, sociales, religiosas del siglo XIX en Colombia, y con la película que lleva el mismo título del texto, cuya directora es Hilda Hidalgo (2009). Se puede observar que estos últimos logran visualizar con mayor profundidad los vínculos entre la literatura y la ciencia, no obstante, parten de un tópico que los mueve con mayor rigor, como lo es el de la ciencia, sin que sea óbice establecer las relaciones con lo humano, la religión y la literatura. Con lo humano porque son muy conscientes de que están estudiando para servirle a la comunidad, a la gente, a las personas; con la religión, porque este es un tema central en la obra de García Márquez; con la literatura porque encontraron un punto de referencia para visualizar la enfermedad desde una visión distinta a la nuestra y era la visión que se tenía en el siglo XIX, cuando la ciencia aún no tenía el desarrollo que tiene hoy día, donde la superstición estaba por encima del desarrollo científico europeo y estadounidense. En este caso podemos decir con Leibrandt $(2013$, p.150) que "la lectura es otro medio de socialización y una posibilidad de adquirir o mejorar en la competencia social y emocional".

En cuanto a la categoría 3, de la relación con las emociones en el programa Nutrición y Dietética, las subcategorías fueron: consumo relacionado con lo emocional y relación del consumo con una población específica. En Medicina, literatura generadora de emociones y la explicación científica de las emociones. Se evidencia, sobre todo en Medicina, que los estudiantes tuvieron una relación fuerte con el texto de García Márquez Del amor y otros demonios, probablemente porque encontraron allí, en medio de una historia de amor, entre una adolescente y un sacerdote joven, una manera distinta de acercarse al tema de la enfermedad, del tratamiento médico en términos de lo ético y lo científico, los dolores del cuerpo y del alma y como futuros médicos cómo responderían ante las demandas de sus pacientes.
En síntesis, la investigación y vivencias con los estudiantes de la Escuela de Nutrición y Dietética y de Medicina de la Universidad de Antioquia deja entrever la importancia que tiene la literatura en carreras dedicadas a la ciencia y que aunque se ha hecho en cursos de corte humanístico de los primeros semestres de ambas, la ausencia de los textos literarios es bastante notoria. Ante la pregunta de investigación ¿ Cómo una experiencia estética con el cuento y la novela permite a un grupo de estudiantes de educación superior identificar el diálogo entre la literatura y las ciencias? Hemos asistido a la experiencia de la lectura de dos grandes obras como lo son las de Esquivel y García Márquez, con quienes los estudiantes establecieron un dialogo directo y pudieron visualizar mundos distintos a los nuestros; es decir, mundos posibles, mundos de ficción que como lo señala Garrido (2011 1, p. 158) "la ficción es el único modo de acceder a uno mismo, ya que solo ella nos permite situarnos simultáneamente dentro y fuera".

Vivir la experiencia de lectura en los inicios del siglo XX con Tita y su familia, la cocina, los sabores mexicanos y europeos, sentir la revolución mexicana en medio del amor, la tristeza, el olvido. Asimismo, habitar las costumbres, los pensamientos y la forma de actuar en una parte de Colombia como lo es Cartagena de Indias, en medio de la presencia de negros, sacerdotes y gente del común del siglo XIX significa tener una experiencia susceptible de ser estética. De igual forma, la hipótesis abductiva: ¿̇una estrategia didáctica fundamentada en la experiencia estética con el cuento y la novela podría contribuir al diálogo entre la literatura y la didáctica universitaria? Como estamos en la búsqueda y la construcción de la estrategia didàctica denominada Cienciatura, pensamos que efectivamente, y con base en las respuestas de los estudiantes de las dos dependencias académicas mencionadas, la estrategia didáctica podrá ser una posibilidad para que en la universidad los profesores de ciencias básicas aborden la literatura como mediación didáctica a fin de trabajar conceptos propios de la ciencia que enseñan. Hemos hallado que autores como Homero, Dante, Ovidio, Cervantes, Voltaire, Tolstoi, Dostoievski, Borges, Calvino, Yourcenar, Allende, Sábato, Carroll, Casares, Paz, Fuentes, Chimal, Pitol, Rulfo, Monsiváis, Arreola, Sor Juana Inés de la Cruz, Volpi, Monterroso, entre otros, han habitado el mundo de la ciencia a través de sus tramas. 
- Aguiar e Silva, V. M. (1986). Teoría de la literatura. Madrid: Gredos.

- Albaladejo, T. (1996). La ficción realista y la ley de máximos semánticos. En: E. Sullá, Teoría de la novela (pp. 297-304). Barcelona: Crítica.

- Arau, A. (Prod. \& Dir.). (1992). Como agua para chocolate [Película]. México: Miramax Films-Arau Films International.

- Argos, J., \& Ezquerra, P. (2013). Entornos hipertextuales y educación. Teoría de la Educación: Educación y Cultura en la Sociedad de la Información, 14(3), 175-190. Recuperado de http://www.redalyc.org/pdf/2010/201029582009.pdf

- Aspiunza, J. (2010). La naturaleza en «Über die bildende Nachahmung des Schönen», de Karl Philipp Moritz. Contrastes: Revista Internacional de Filosofía, 15, 25-42. Recuperado de http://www.uma.es/contrastes/pdfs/015/ContrastesXV-02.pdf

- Barthes, R. (1984). S/Z. Buenos Aires: Siglo XXI.

- Baumgarten, A. G. (1975). Reflexiones filosóficas acerca de la poesía. Buenos Aires: Aguilar.

- Borges, J. L. (2000). El Aleph. Buenos Aires: Alianza Editorial.

- Bruner, J. (1987). Realidad mental y mundos posibles: Los actos de la imaginación que dan sentido a la experiencia. Barcelona: Gedisa.

- Cabot, M. (1999). La importancia de los estudios estéticos del siglo XVIII. Recuperado de http://www.mateucabot.net/pdf/ cabot_importancia.pdf.

- Cachapuz, A. F. (2007). Arte y ciencia: ¿̇ué papel juegan en la educación en ciencias? Eureka: Sobre Enseñanza y Divulgación de las Ciencias, 4(2), 287-294. Recuperado de http://venus.uca.es/eureka/revista/Volumen4/Numero_4_2/ Vol_4_Num_2.htm

- Capanna, P. (1966). El sentido de la ciencia-ficción. Buenos Aires: Columba. Recuperado de http://inabima.gob.do/descargas/ bibliotecaFAlL/Autores\%20Extranjeros/C/Capanna,\%2.

- Cortázar, J. (1985). Rayuela. Buenos Aires: Círculo de Lectores.

- Del Valle, J. (2011). La dignidad de la imaginación. Alexander Baumgarten y el contexto de nacimiento de la estética. Areté. Revista de Filosofía, 23(2), 303-328. Recuperado de http:// www.scielo.org.pe/scielo.php?script=sci_arttext\&pid=\$1016913X2011000200004\&lng=es\&nrm=iso

- Djerassi, C. (2011). La historia de la obra teatral "Oxígeno». Ciència i literatura, del paper a l'escenari. Métode, 69, 97-102.
Recuperado de http://metode.cat/es/revistas/monografics/ afinidades-electivas/la-historia-de-lobra-

- Dolezel, L. (1999). Heterocósmicas: Ficción y mundos posibles (Perspectivas). España: Arco/Libros.

- Eco, U. (1993). Lector in fabula: La cooperación interpretativa en el texto narrativo. Barcelona: Lumen.

- Esquivel, L. (1993). Como agua para chocolate. Barcelona: RBA.

- Farina, C. (2005). Arte, cuerpo y subjetividad. Estética de la formación y pedagogia de las afecciones (Tesis doctoral, Universitat de Barcelona). Recuperada de http://biblioteca. universia.net/html_bura/ficha/params/title/arte-cuerposubjetividad-experiencia-estetica-pedagogica/id/55252878. html

- García García, J. J., \& González Agudelo, E. M. (2007). Entre la literatura y las ciencias experimentales: Hacia una mirada estética para el desarrollo didáctico de una cultura científica. Uni-pluri/versidad, 7(1), 39-45. Recuperado de http:// aprendeenlinea.udea.edu.co/revistas/index.php/unip/article/ viewFile/11921/

- García Márquez, G. (1994). Del amor y otros demonios. Bogotá: Círculo de Lectores.

- Garrido Domínguez, A. (2011). Narración y ficción: Literatura e invención de mundos. Madrid: Iberoamericana/Vervuert.

- Giannetti, C. (2002). Estética digital: Sintopía del arte, la ciencia y la tecnología. Barcelona: ACC L'Angelot. Recuperado de http://artmetamedia.net/pdf/4Giannetti_EsteticaDigitalES.pdf

- Goleman, D. (1996). Inteligencia emocional. Barcelona: Kairós.

- González Agudelo, E. M. (2011). Sobre la experiencia hermenéutica o acerca de otra posibilidad para la construcción del conocimiento. Discusiones Filosóficas, 12(18), 125-143. Recuperado de http://www.scielo.org.co/scielo. php?script=sci_arttext\&pid=S0124-612

- Henao, R. D. (2014). La razonabilidad estética como proceso interhumano y abductivo desde "Un descenso al Maelström". Enunciación, 19(1), 53-64. doi: http://dx.doi.org/10.14483/ udistrital.jour.enunc.2014.1.a04

- Hidalgo, H. (Dir.). (2009). Del amor y otros demonios [Película]. Costa-Rica-Colombia-México: CMO Producciones-Alicia FilmsCacerola Films, S. A. de C. V.

- Huxley, A. (1979). Un mundo feliz. Bogotá: Plaza \& Janés. 
- Ianni, O. (2006). Teorías de la globalización (7ª ed.). México: Siglo XXI. Recuperado de https://books.google.com.mx/

- Iser, W. (2005). Rutas de la interpretación. México: Fondo de Cultura Económica.

- Jauss, H. R. (1992). Experiencia estética y hermenéutica literaria: Ensayos en el campo de la experiencia estética. Madrid: Santillana.

(2000). La historia de la literatura como provocación. Barcelona: Península. (2002). Pequeña apología de la experiencia estética. Barcelona: Paidós.

- Kant, I. (2010). Crítica del juicio. Madrid: Gredos.

- Labrada, M. A. (1983). Estética y filosofía del arte: Hacia una delimitación conceptual. Anuario Filosófico, 16(2), 67-80. Recuperado de http://dadun.unav.edu/handle/10171/2173

- Landow, G. (1995). Hipertexto: La convergencia de la teoría crítica contemporánea y la tecnología. Barcelona: Paidós.

- Larrosa, J. (2003). La experiencia de la lectura: Estudios sobre literatura y formación. Barcelona: Laertes.

- Leibrandt, I. (2012). La literatura juvenil histórica como medio para la construcción de la identidad. Impossibilia. Revista Internacional de Estudios Literarios, 4, 184-200. Recuperado de http://ojs.impossibilia.org/index.php/impossibilia/article/ view/37/39

(2013). Fomentar la competencia emocional a través de la LIJ. Lenguaje y textos. Revista de la sociedad española de didàctica de la lengua y la literatura, 38, 149-157. (2016). La formación estético emocional a través de la literatura. Granada: Comares.
- Maturana, H., \& Varela, F. (1998). De máquinas y seres vivos. Autopoiesis: La organización de lo vivo (5a. ed.). Santiago de Chile: Editorial Universitaria.

- Montes, G. (1999). De lo que sucedió cuando la lengua emigró de la boca. Lectura y Vida. Revista Latinoamericana de Lectura, 20(3), 2-10.

- Moreno Torres, M., \& Carvajal Córdoba, E. (2015). Bases de una estrategia didáctica para la formación de profesores investigadores. Bogotá: Aula de Humanidades.

- Moritz, K. P. (2004). Propuesta para la unificación de todas las bellas artes y las ciencias bajo el concepto de lo perfecto en sí mismo. En Burguera, M. L. (Ed.), Textos clásicos de teoría de la literatura. Madrid: Cátedra.

- Nubiola, J. (febrero-abril, 2001). La abducción o lógica de la sorpresa. Razón y Palabra, 21. Recuperado de http://www. razonypalabra.org.mx/anteriores/n21/21_jnubiola.html

- Rueda Ortiz, R. (2007). Pensar una pedagogía del hipertexto. Una teoría de la deconstrucción y la complejidad. Barcelona: Anthropos Editorial. Quintana Ramírez, A. (2007). Ellos vienen con el chip incorporado: Aproximación a la cultura informática escolar (2a . ed.). Bogotá: Instituto para la Investigación Educativa y el Desarrollo Pedagógico.

- Téllez, M. (2004). Entre marcas y señales: Reabrir la pregunta por la razón de ser de la universidad. En H. Casanova, \& C. Lozano (Eds.), Educación, universidad y sociedad: El vínculo crítico (pp. 216-238). Barcelona: Universidad de Barcelona/ Universidad Nacional Autónoma de México. Recuperado de http://www.mec.maestrias.unach.mx/images/tablas/4/mingo. pdf

- Vargas Guillén, G., y Rueda Ortiz, R. (1996). La pedagogía ante la tecnología como estructura del mundo de la vida. Nómadas, 5. 